\title{
Color translucent head-up display based on a segmented Fourier hologram
}

\author{
Jaroslaw Suszek, ${ }^{*}$ Maciej Sypek, Andrzej Siemion, Agnieszka Fajst, Michal Makowski and Andrzej Kolodziejczyk \\ Faculty of Physics, Warsaw University of Technology, Koszykowa 75, 00-662 Warsaw
}

Received September 10, 2009; accepted September 28, 2009; published September 30, 2009

\begin{abstract}
The paper presents the numerical and experimental results of reconstructing images from a spatially segmented Fourier hologram with additional carrier frequencies, acting as a translucent head-up display (HUD). Successful reconstructions of colorful images visible at the infinity were achieved. The design procedure is described and some limitations of the method are discussed, like the appearance of ghost images. Exemplary results from numerical and real experiments are given. In order to obtain color images three beams of primary colors were successfully utilized to create a color mixture.
\end{abstract}

In this paper we present preliminary experimental results of reconstructing full-color images from a translucent holographic HUD-type display. Such a display could potentially be applied in motor industry [1] for presenting crucial information to a driver without the need of taking the eyes off the road. This issue has been under a constant worldwide investigation [2].

The necessity of repeated accommodation from close objects, like a dashboard, to far distances, like road signs and vice-versa causes eye-strains and tiredness. Furthermore, this shift of focal length lasts approximately half a second, thus decreasing safety while driving, especially at high speeds. A driver looking through the proposed display would not have to accommodate at all, since the images are presented at the infinity. Additionally, in the case of helmet-mounted displays for motorcycles precise alignment of the display against the eye is possible. Therefore the symbols are always visible regardless of the current position of the driver's head, which could potentially be a problem in the case of cars.

The presented display is in fact a static phase-only binary Fourier hologram reconstructed with laser beams at varying angle of incidence and varying wavelength, or color. Eight different asymmetric symbols were encoded using a spatial segmentation technique [3] with the use of variable carrier frequencies. A chessboard-like segmentation function was used. Both the orientations and periods of the saw-blade diffractive gratings corresponding to the carrier frequencies were chosen in order to achieve a switching of displayed symbols by simply altering the incidence angle of the illumination. The laser beams were transformed into plane waves of three primary colors: red, green and blue.

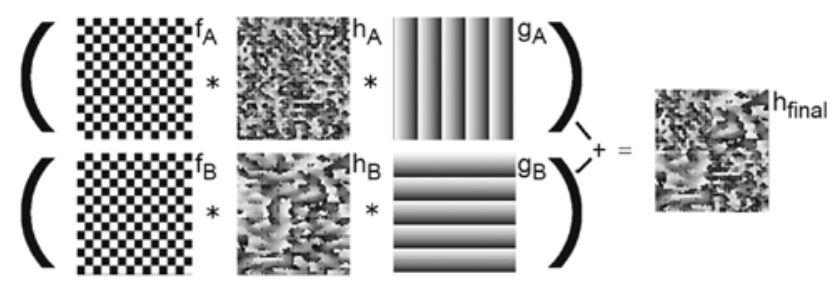

Fig. 1. The basic idea of the design of a HUD-like hologram, the transmittances are magnified for clarity.

The algorithm of creating the phase transmittance of a HUD hologram for two different symbols is illustrated in Fig. 1. Phase distributions marked as $h_{A}$ and $h_{B}$ were precalculated using the IFTA [4] (Iterative Fourier Transform Algorithm). This optimization was performed to suppress the speckles and to increase the contrast of reconstructed images. The obtained phase patterns were multiplied by appropriate segmentation functions, noted as $f_{A}$ and $f_{B}$ in order to merge them into a single hologram later on. In the next step the transmittances were multiplied by phases of saw-blade gratings noted as $\mathrm{g}_{\mathrm{A}}$ and $g_{B}$ in order to achieve the effect of matching a particular symbol with a particular incidence angle of the illumination. Finally, the transmittances A and B are added and directly binarised. Due to the used carrier frequencies the additional ghost images formed in the $-1^{\text {st }}$ order of diffraction are shifted outside of the viewing range. Thanks to this, in a single binary structure we have encoded a set of asymmetric symbols. An exemplary reconstruction in a Fourier configuration from a hologram with two symbols encoded is presented in Fig. 2.

An unavoidable consequence of using regular chessboard-like segmentation function is the appearance of additional ghost multiplications of the image, as shown in Fig. 3.

\footnotetext{
*E-mail: suchy@if.pw.edu.pl
} 


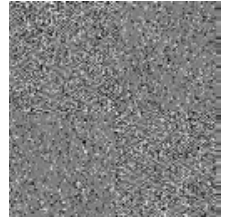

a)

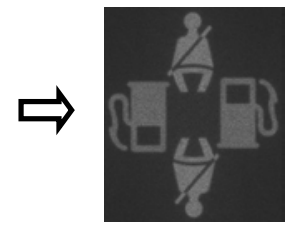

b)

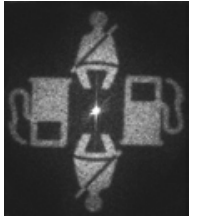

c)
Fig. 2. The Fourier hologram with spatial segmentation and carrier frequencies added for the case of two symbols: a) magnified central region of the phase; b) numerical reconstruction; c) experimental reconstruction captured in the focal plane of a converging wave.

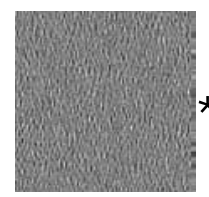

a)

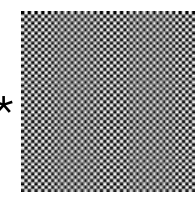

b)

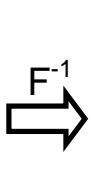

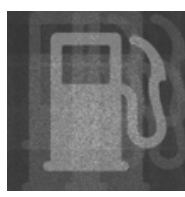

C)

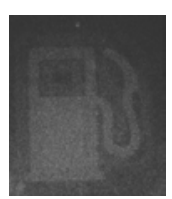

d)
Fig. 3. The source of multiplicated ghost images: a) phase transmittance of a hologram; b) segmentation function; c) numerical reconstruction; d) experimental reconstruction.

Nevertheless by choosing an optimal period of the segmentation function one can achieve a reasonable trade-off.

In the experiment presented above a segmentation period of $1 \mathrm{~mm}$ was used with a size of the whole hologram equal to $10.24 \mathrm{~mm}$ by $10.24 \mathrm{~mm}$ (designed on a matrix of 1024 by 1024 points with a sampling of $10 \mu \mathrm{m}$ ).

The experimental results presented below were gathered from a binary-phase element made in the electron-beam lithography technique. The resolution was 16384 by 16384 points with a sampling of $1 \mu \mathrm{m}$. The structure had eight asymmetric symbols encoded.

The color images were reconstructed by a proper illumination with plane waves formed by a He-Ne laser (red, $\lambda=632.8 \mathrm{~nm}$ ), a frequency-doubled Nd-YAG (green, $532 \mathrm{~nm}$ ) and an Argon laser (blue, $488 \mathrm{~nm}$ ). The carrier frequencies were chosen to match the mentioned wavelengths in order to obtain the visible effect of color mixing. Fig.4. presents an experimental result of reconstructing one of the eight symbols with a red beam, a green beam and two beams simultaneously. Hence by a proper mixture of illumination with different beams at different angles one can obtain even a wider range of colors.

By a simultaneous illumination with three beams a white color is easily achievable, as seen in Fig. 5.

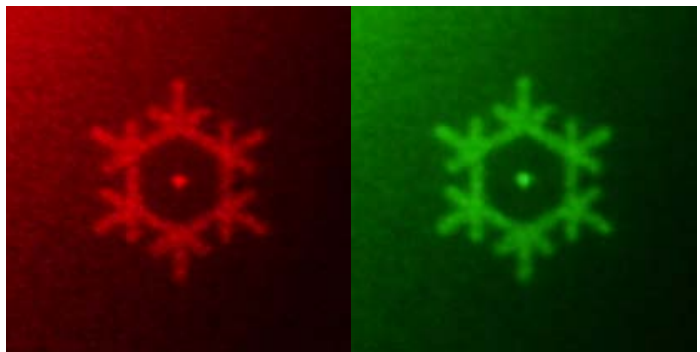

a)

b)

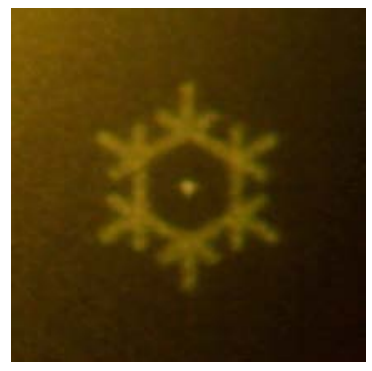

C)

Fig. 4. The reconstruction of a "snow" symbol with: a) 632,8 nm beam; b) $532 \mathrm{~nm}$ beam; c) both beams.

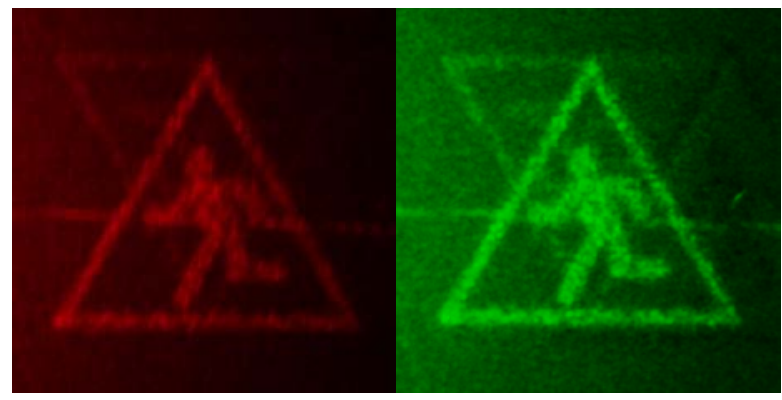

a)

b)

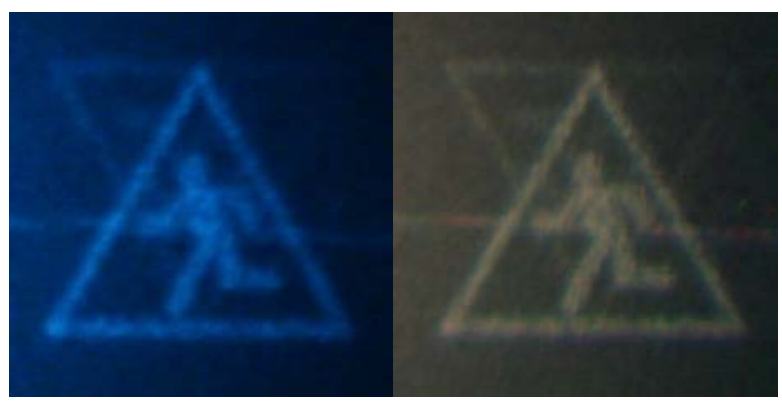

C)

d)

Fig. 5. The reconstruction of a "pedestrian" symbol with: a) 632,8 nm beam; b) $532 \mathrm{~nm}$ beam; c) $488 \mathrm{~nm}$ beam; c) three beams combined. 
In the case of practical applications, the laser illumination can be replaced with a set of color LED diodes. This should completely eliminate the speckles in the obtained images. On the other side, in this case the wavefront curvature from LEDs would have to be compensated.

The authors would like to thank HOLOEYE Photonics AG for valuable support. This work has been supported by the European Union in the framework of European Social Fund through the Warsaw University of Technology Development Programme.

\section{References}

[1] A. Ramsbottom, S. Sergeant, D. Sheel, Proc. SPIE 1667, 146-164 (1992).

[2] T. Matsumoto, S. Eguchi, F. Yamagishi, M. Nakashima, Proc. SPIE 2652, 341-344 (1996).

[3] J. Suszek, M. Makowski, M. Sypek, A. Siemion, A. Kolodziejczyk, Appl. Opt. 48, 270-275 (2009).

[4] R. W. Gerchberg, W. O. Saxton, Optik 35, 237-246 (1972). 